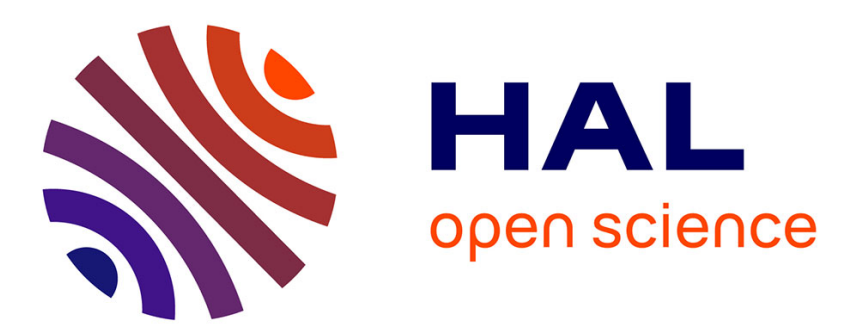

\title{
Recent evolutions in low phase noise microwave sources and related problems of noise modeling
}

Olivier Llopis, Sébastien Gribaldo, Cédric Chambon, Bertrand Onillon, Jean-Guy Tartarin, Laurent Escotte

\section{- To cite this version:}

Olivier Llopis, Sébastien Gribaldo, Cédric Chambon, Bertrand Onillon, Jean-Guy Tartarin, et al.. Recent evolutions in low phase noise microwave sources and related problems of noise modeling. 19th International Conference on Noise and Fluctuations - ICNF2007, Sep 2007, Tokyo, Japan. pp.353-358. hal-00177027

\section{HAL Id: hal-00177027 \\ https://hal.science/hal-00177027}

Submitted on 5 Oct 2007

HAL is a multi-disciplinary open access archive for the deposit and dissemination of scientific research documents, whether they are published or not. The documents may come from teaching and research institutions in France or abroad, or from public or private research centers.
L'archive ouverte pluridisciplinaire HAL, est destinée au dépôt et à la diffusion de documents scientifiques de niveau recherche, publiés ou non, émanant des établissements d'enseignement et de recherche français ou étrangers, des laboratoires publics ou privés. 


\title{
Recent evolutions in low phase noise microwave sources and related problems of noise modeling
}

\author{
O. Llopis, S. Gribaldo, C. Chambon, B. Onillon, J.G. Tartarin, L. Escotte \\ LAAS-CNRS, Toulouse University, 7 av. du Colonel Roche, 31077 Toulouse, France \\ llopis@laas.fr
}

\begin{abstract}
The evolution of spectrally pure microwave sources is described, together with the problem of modeling the spectral shape of these signals. The noise sources interaction with the intrinsic nonlinear behavior of the oscillator is pointed out, and various solutions are proposed. Original techniques are shown to investigate on the phase noise modeling directly at the transistor level. Then, the problem of noise in some new approaches for frequency generation, in which other noise sources than the transistor noise contributes to the oscillator noise, is described.
\end{abstract}

Keywords: microwave oscillator, frequency generation, phase noise, nonlinear noise PACS: $43.58 . \mathrm{Wc}$

\section{INTRODUCTION}

Spectrally pure frequency generation is necessary in many applications. Precision timing, as an example, is directly related to the source phase noise. More generally, in many physics applications, the frequency is the parameter on which the highest constraints are put on. However, the strongest economical challenge in this field is today for telecommunications applications. The phase noise of the oscillator is indeed superimposed directly to the signal when this signal is demodulated or simply converted in frequency. Thus, the oscillator noise close to the carrier becomes the limit of the signal to noise ratio at the receiver output. Of course, different types of oscillators are used in physics or telecommunications applications. Physicists are mainly seeking performance meanwhile the telecommunications industry needs small size and low cost systems, which means a high level of integration. However, the theoretical description of the noise mechanisms in these systems is very similar, even if the frequency reference element (the resonator) is different. Another application field, which could be located between the two previous ones, is the development of highly sensitive radars or telemeters. This is of high interest for military purpose, but not only. In these applications, both high performance and small size are needed, which is of course difficult to achieve.

Because of these different application fields, research on high spectral purity oscillators is performed in different scientific communities : the time and frequency community first ; secondly the microwave community, which drives research for both telecommunications and military applications ; then the circuit community, which is also related to the telecommunications field, and which is focused on integrated sources. The last community is of course the one of noise. Indeed, the investigations 
on phase noise, and particularly the pioneering work performed in the microwave field [1-4], have revealed some theoretical problems related to the description of noise under nonlinear conditions. It was thus mandatory to think again the transistor noise modeling, which had been developed for linear applications, and to adapt this modeling to the description of a nonlinear problem. Of course, the device modeling is only one part of the problem, the other one being the development of powerful CAD tools, able to compute the phase noise from these models in an complex circuit. Such tools have been developed in the 1990s [2], and the problem of the noise transposition in a nonlinear circuit between the different harmonics is now well described by these approaches. However, the effect of the nonlinear signal on the noise source itself is still a disputed subject. This problem will be discussed in this paper.

Describing the oscillator phase noise through the behavior of the transistor noise under nonlinear conditions is valid, providing the other elements of the oscillator do not contribute too much to the oscillator noise. In this case, it is possible to improve the oscillator performance finding out the appropriate transistor load at each harmonics which minimizes the overall output phase noise. This approach can be efficient even if the complete noise generation process at the transistor level is not completely understood. However, for some applications it is mandatory to be able to simulate the oscillator phase noise with a high precision (because the circuit cannot be tuned after being realized). In this case, a model able to precisely describe the noise under nonlinear conditions is required.

The problem discussed in the above paragraph is the one of any microwave circuit using only passive elements for frequency reference: integrated LC oscillators and VCOs, dielectric resonator or coaxial resonator oscillators, sapphire oscillators... However, if the resonator itself is not passive, it may be the predominant contribution to the output phase noise. This is the case of acoustic wave resonators, which feature a $1 / f$ fluctuation of their resonant frequency. Also, very recently, it has been proposed to use optical delay lines as the frequency reference elements of microwave oscillators. However, in this case also, the signal conversion to optics add its noise contribution, which must be evaluated.

\section{MODELING OF OSCILLATORS WITH PASSIVE RESONATORS}

As described in Leeson's paper [5], an oscillator is a feedback loop with a phase fluctuating amplifier and a resonator in the feedback. The amplifier phase noise is directly converted through the loop effect into the oscillator's frequency noise. Investigating the phase noise of the amplifier is simpler than investigating on the complete oscillator, because : 1) there is no phase loop condition, so the measurement can be performed in well defined conditions 2) the measurement can be performed on a large RF power range 3) it is easier and faster to simulate the performance of a driven circuit than an autonomous circuit 4) It is essential to investigate the noise conversion processes independently of the surrounding circuit. To this purpose, a measurement bench has been developed [6] which is able to characterize this parameter, either on an already designed amplifier or on a single transistor on which both the RF and LF loads are controlled. 


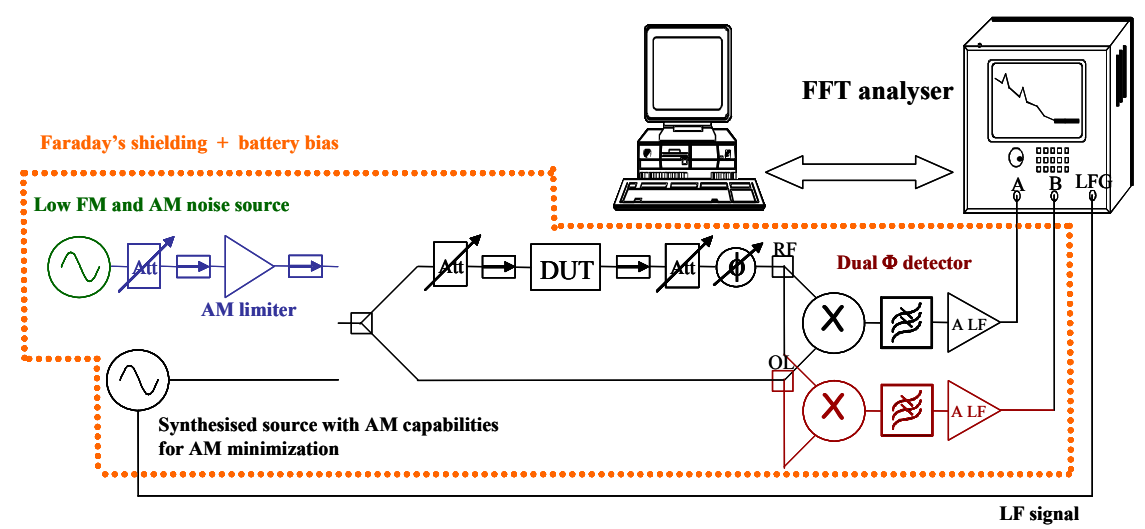

FIGURE 1. Residual phase noise (or amplifier phase noise) measurement bench, which is able to perform phase noise measurements on the 1 to $18 \mathrm{GHz}$ range (using different low FM noise sources)

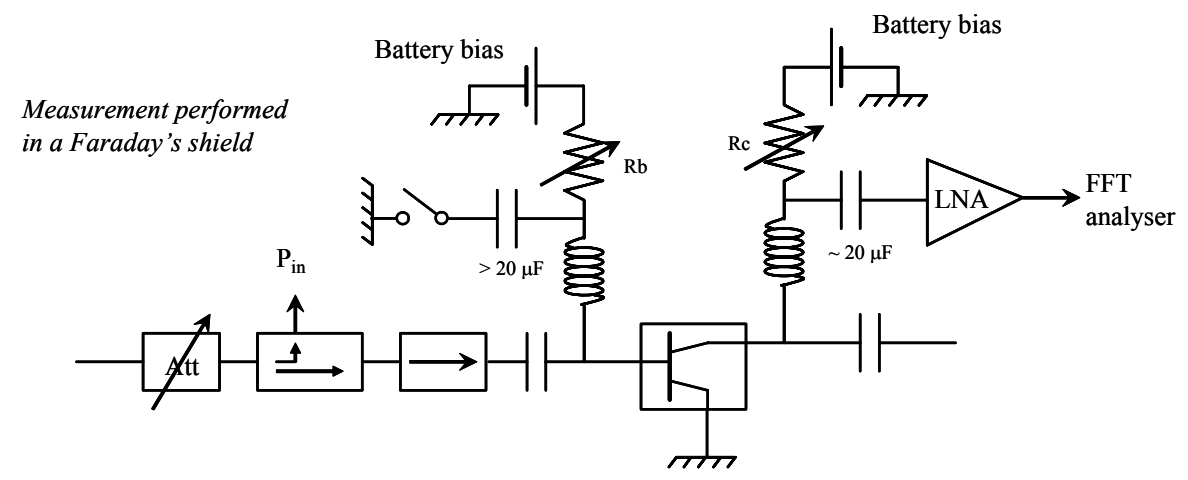

FIGURE 2. Details of the part of the experiment used to investigate on the device LF noise under nonlinear signal conditions.

The goal of these measurements is to study the correlation between the transistor low frequency (LF) noise and the transistor phase noise. The transistor LF noise should therefore be measured in the same operating conditions i.e. submitted to the nonlinear signal. This is performed on the same measurement bench, adding an output on the device bias Tees and a control of the device LF loads (Figure 2).

An example of the data obtained with this measurement bench is given in Figure 3. The microwave power at $3.5 \mathrm{GHz}$ is smoothly increasing from the linear regime up to $4 \mathrm{~dB}$ compression. The change in the spectral shape between the linear behavior and the nonlinear behavior is strong, and the noise in the nonlinear behavior can hardly be predicted using the linear (or quiescent) data. However, there is obviously a strong correlation between the LF noise and the phase noise, which means that a global modeling able to describe simultaneously these too spectrum sets could be extracted. The model which had been proposed for this device [7] involved to main LF noise sources, one of which (a g.r. noise source) taking advantage on the other one when the device was driven far in the nonlinear state. In this model, the nonlinear behavior of this g.r. noise source had been described through the association of this noise source with a nonlinear element, which was introduced in the device electrical model mainly to fit the measurement rather than because it had a physical meaning. Other authors argued that the noise source itself could be treated as a nonlinear element. This has 
been proposed as early as the first experiments [1], and the more recent papers on this subject seems to be in favor of this approach [8-11]. However, an equivalent circuit modeling remains a rough approximation of the transistor behavior, contrarily to physical modeling. A good model, at this level of approximation, is simply a model which is able to describe the measurement, at various input power levels or for different transistor load. So if good results can be obtained with a noise source instantaneously sensitive to the microwave signal, and there are some physical arguments that the noise could behave in this way, this approach should become the standard approach to model the noise in nonlinear circuits.
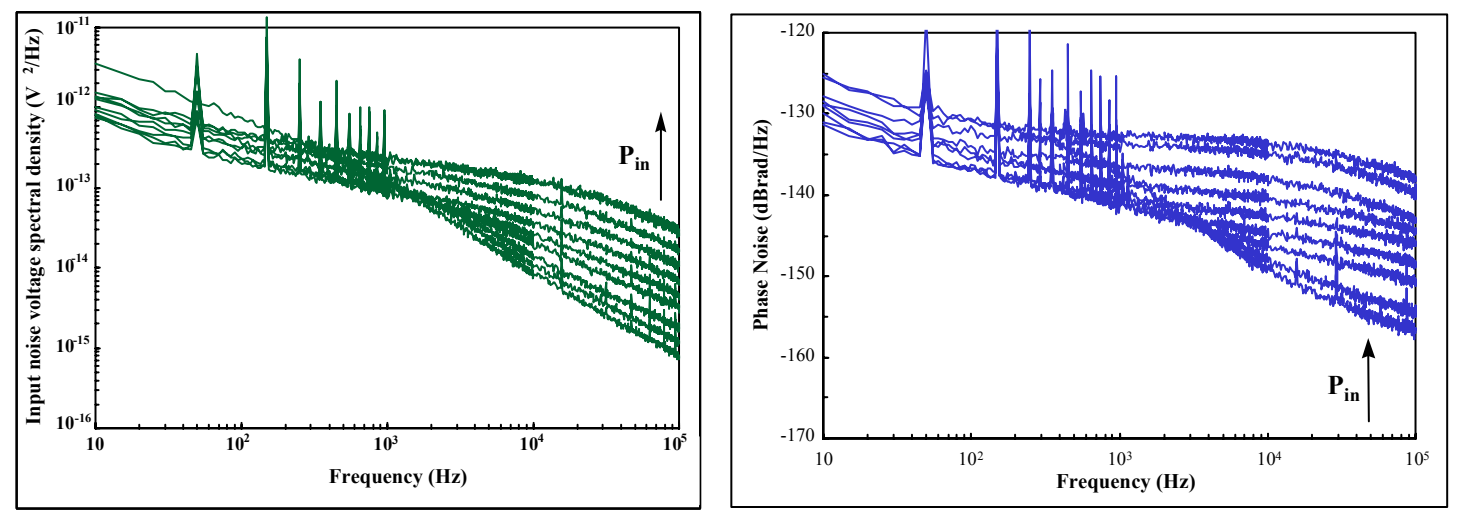

FIGURE 3. Example of data obtained on a FET device [7] ; left : equivalent input voltage noise spectral density at different microwave power levels $\left(\mathrm{P}_{\mathrm{in}}\right)$; right : phase noise at $3.5 \mathrm{GHz}$, the transistor being loaded onto $50 \Omega$ at $\mathrm{RF}$, for the same range in $\mathrm{RF}$ power $\left(\mathrm{P}_{\text {in }}\right)$

We have thus used this approach to model the phase noise generated by a SiGe HBT. We will not describe in details this model here, because it is presented in a focused paper in these proceedings [12]. It is an improvement of a simpler model which was using external noise sources parameterized by the microwave power [13]. This technique, which uses power dependant external LF noise sources, is very efficient, but it requires that the noise parameters are measured on an amplifier which is close to the one which will be used in the final oscillator circuit. It allows anyway some phase noise optimization, and as proven its efficiency on the design of high spectral purity sources [14]. The new model is no more based on an extrinsic parameter. On the contrary, the nonlinear behavior arises from an instantaneous dependence of the collector current noise spectral density $S_{i c}$ to the RF signal. $S_{i c}$ is supposed to be composed of a constant coefficient and of a second term proportional to $\dot{i}_{\mathrm{c}}{ }^{2}(\mathrm{t})$. In the commercial circuit design software (Agilent ADS) used to simulate the phase noise, this noise source is considered just like a classical nonlinear element, using a symbolically defined device. The parameters of this new type of nonlinearity are extracted from the measurement of the equivalent input LF noise at different RF power levels [12]. Other authors use to this purpose multi-bias noise measurements $[10,11]$, but we think that the measurement of noise under nonlinear conditions is closer to the final behavior of the transistor and is more reliable for the extraction of the noise nonlinear behavior. 
The above discussion is focused on the effect on phase noise of the device LF noise. However, phase noise is the fluctuation of a high frequency signal, an even if close to the carrier this fluctuation is dominated by the LF fluctuations ( $1 / \mathrm{f}$ or $\mathrm{g} . \mathrm{r}$. noise), far from the carrier it can be the result of the superposition of microwave noise. These two behaviors are described as multiplicative (or conversion) noise and additive noise [15]. The additive noise is a carrier to noise ratio, and is thus almost inversely proportional to the RF signal level. On the contrary, the multiplicative noise comes from a modulation or conversion process, and follows the signal level. The microwave noise has an advantage compare to the $1 / \mathrm{f}$ noise : it is generally well localized in the transistor model (thermal noise of the resistances, schottky noise in the junctions). However, it is also affected by the nonlinear behavior, and the classical approach which involves the transistor noise figure is no more valid, unless a nonlinear noise figure can be simulated or measured [15]. This last noise contribution can be simulated using modern CAD tools or, also, can be investigated experimentally in a relatively similar way which is used to find the minimum noise figure of an amplifier. A focused paper on such an approach can be found in this volume [16].

\section{NOISE PROBLEMS IN MICROWAVE SOURCES USING ACTIVE FREQUENCY REFERENCE ELEMENTS}

The evolution of microwave sources has introduced recently some new problems of noise modeling. Firstly, the telecommunications industry needs today highly integrated high quality sources, and has succeeded very recently in this field thanks to a new device : the integrated thin film bulk acoustic wave resonator. This resonator is an integrated version of the quartz resonator, and it is able to provide a $\mathrm{Q}$ factor of $10^{2}$ to $10^{3}$ in the low microwave range $(1$ to $5 \mathrm{GHz}$ ). Integrated oscillators based on this resonator feature a much better performance than classical LC based VCOs [17]. However, the performance of these sources is limited by an intrinsic $1 / \mathrm{f}$ fluctuation of the resonator frequency, which appears to be higher than the effect of the transistor phase noise [18]. Up to the author's knowledge, no modeling approach has been proposed for this frequency fluctuation, or at least no modeling approach which could be embedded in a circuit simulator. Investigations on this type of noise, and on integrated acoustic wave resonator oscillators, are the key of success for the development of the next generation of small volume microwave sources.

On the other side of oscillators applications, where high performance is the main goal, optical techniques are today foresee to design very high spectral purity microwave sources. In this case, the microwave signal is transferred to the optical range thanks to an optical carrier. Once in the optical domain, it is possible to take benefit of the ultra low losses of the optical delay lines which can trap the signal in a few kilometers line with almost no attenuation, except the one due to the microwave to optical (MO) and optical to microwave (OM) conversions. The equivalent $\mathrm{Q}$ factor is very high, and high performance oscillators [19] or phase noise measurement benches [20] have been realized with this technique. One of the drawbacks is in the size required for a few kilometers fiber optics delay line. To overcome this problem, it has been proposed to replace the line by an optical resonator, but this is difficult and still under study. One of the problems of these systems is the carrier to noise degradation 
due to the $\mathrm{MO}$ and $\mathrm{OM}$ conversions. This carrier to noise ratio is determined by the efficiency of the optical modulation or detection, and by the laser relative intensity noise (RIN). The last parameter may also influence the close to carrier phase noise because of the laser $1 / \mathrm{f}$ RIN. Simulating the overall noise behavior of such a system is difficult, and up to now only analytical approaches have been proposed. The goal is now to develop models and CAD tools able to manage the noise simultaneously in RF and optical devices, in order to optimize these new hybrid systems.

\section{CONCLUSION}

Reducing the phase noise of a high frequency oscillator is one of today's more difficult modeling problems in electronics. This is because it requires an accurate description of the noise under nonlinear behavior. Experimental approaches have been described to check the validity of the various modeling approaches, and a focus is made on models involving nonlinear noise sources. Finally, the problem of noise studies of more complex sources involving resonances which are not entirely of electrical type is pointed out. All these systems still requires investigations in the field of noise modeling.

\section{REFERENCES}

1. H.J. Siweris, B. Schiek, "A GaAs FET oscillator noise model with a periodically driven noise source", 1986 EuMC Proceedings, pp. 681-686.

2. V. Rizzoli, F. Mastri, D. Masotti, IEEE Trans. on MTT, vol 42, n 5, pp. 807-819, 1994.

3. J. Verdier, O. Llopis, R. Plana, J. Graffeuil, IEEE Trans. Microwave Theory Tech, Vol. 44, $\mathrm{N}^{\circ}$ 8, pp. 14611471, August 1996.

4. O. Llopis, et al, "Nonlinear noise modeling of a PHEMT device through residual phase noise and low frequency noise measurements," Proc. of the IEEE MTT Symposium, pp. 831-834, May 2001.

5. D. B. Leeson, Proc. of the IEEE, vol 54, n ${ }^{\circ}$ 2, Feb. 1966, pp. 329-330.

6. G. Cibiel, M. Régis, E. Tournier, O. Llopis, IEEE trans. on Ultrasonics Ferroelect. and Freq. Control, vol. 49, $\mathrm{n}^{\circ}$ 6, Juin 2002, pp. 784-788.

7. O. Llopis, et al, "Nonlinear noise modeling of a PHEMT device through residual phase noise and low frequency noise measurements," Proc. of the IEEE MTT Symposium, pp. 831-834, May 2001.

8. F. Bonani, S. D. Guerrieri, G. Ghione, IEEE Trans. on ED, vol 49, n 9, sept. 2002, pp. 1640-1647.

9. M. Borgarino, C. Florian, P.A. Traverso, F. Filicori, IEEE Trans. on ED, vol 53, n 10, oct. 2006, pp. 26032609.

10. J.C. Nallatamby et al., IEEE Trans. on MTT, vol 53, n 5, May 2005, pp. 1601-1612.

11. M. Rudolph et al., IEEE Trans. on MTT, vol 54, $\mathrm{N}^{\circ}$ 7, july 2006, pp. 2954-2961.

12. S. Gribaldo, O. Llopis, "SiGe HBT nonlinear phase noise modeling" ICNF 2007, this volume

13. S. Gribaldo, G. Cibiel, O. Llopis, and J. Graffeuil, "Nonlinear noise in SiGe bipolar devices and its impact on radio-frequency amplifier phase noise," Proc. ICNF 2005, Salamanca (Spain), 2005, pp. 513-516.

14. G. Cibiel, et al, IEEE Transactions on Ultrasonics, Ferroelectrics, and Frequency Control, Vol. 51, No. 1, pp. 33-41, January 2004.

15. G. Cibiel, et al, IEEE Transactions on Microwave Theory and Techniques, Vol. 52, pp 183-190, January 2004.

16. C. Chambon, L. Escotte, ICNF 2007, this volume

17. M. Aissi et al., "A 5.4 GHz 0.35um BiCMOS FBAR resonator oscillator in above-IC technology" 2006 IEEE International Solid-State Circuits Conference (ISSCC), San Francisco, pp.316-317.

18. S. Gribaldo et al., IEEE Transactions on UFFC, vol 53, n 11, nov. 2006, pp. 1982-1987.

19. X.S. Yao, L. Maleki, D. Eliyahu, "Progress in the optoelectronic oscillator - a ten year anniversary review", 2004 IEEE Microwave Theory and Tech. Symp. Digest, pp. 287-290.

20. B. Onillon, S. Constant, O. Llopis, "Optical links for ultra-low phase noise microwave oscillators measurement”, Proc. of the IEEE Int. Freq. Control Symposium, Vancouver, août 2005, pp. 545-550. 